\title{
Models for X-Ray Emission from Isolated Pulsars
}

\author{
F. Y.-H. Wang, M. Ruderman, J. P. Halpern, and T. Zhu \\ Columbia Astrophysics Laboratory, Columbia University, \\ 550 West 120th Street, New York, NY 10027 \\ fyw@orphee.phys.columbia.edu
}

\begin{abstract}
A model is proposed for the observed combination of power-law and thermal emission of $\mathrm{keV} \mathrm{X}$-rays from rotationally powered pulsars. For $\gamma$-ray pulsars with accelerators very many stellar radii above the neutron star surface, 100 $\mathrm{MeV}$ curvature $\gamma$-rays from $e^{-}$or $e^{+}$flowing starward out of such accelerators are converted to $e^{ \pm}$pairs on closed field lines all around the star. These pairs strongly affect X-ray emission from near the star in two ways. (1) The pairs are a source of synchrotron emission immediately following their creation in regions where $B \sim 10^{10} \mathrm{G}$. This emission, in the photon energy range $0.1 \mathrm{keV} \lesssim E_{\mathrm{X}} \lesssim$ $5 \mathrm{MeV}$, has a power-law spectrum with energy index 0.5 and X-ray luminosity that depends on the backflow current, and is typically $\sim 10^{33} \mathrm{ergs} \mathrm{s}^{-1}$.

The pairs ultimately form a cyclotron resonance "blanket" surrounding the star except for two holes along the open field line bundles which pass through it. In such a blanket the gravitational pull on $e^{ \pm}$pairs toward the star is balanced by the hugely amplified push of outflowing surface emitted X-rays wherever cyclotron resonance occurs. Because of it the neutron star is surrounded by a leaky "hohlraum" of hot blackbody radiation with two small holes, which prevents direct X-ray observation of a heated polar cap of a $\gamma$-ray pulsar. Weakly spin-modulated radiation from the blanket together with more strongly spin-modulated radiation from the holes through it would then dominate observed low energy (0.1-10 keV) emission. For non- $\gamma$-ray pulsars, in which no such accelerators with their accompanying extreme relativistic backflow toward the star are expected, optically thick $e^{ \pm}$resonance blankets should not form (except in special cases very close to the open field line bundle). From such pulsars blackbody radiation from both the warm stellar surface and the heated polar caps should be directly observable. In these pulsars, details of the surface magnetic field evolution, especially of polar cap areas, become relevant to observations. The models are compared to X-ray data from Geminga, PSR 1055-52, PSR 0656+14, PSR 1929+10, and PSR 0950+08.
\end{abstract}


Subject headings: pulsars: individual (Geminga, PSR 1055-52, PSR 0656+14, PSR 1929+10, PSR 0950+08) — stars: neutron — X-rays: stars

\section{Introduction}

X-ray emission at keV energies from pulsars may come from several sources: (a) thermal cooling from the entire stellar surface of deep internal heating source (e.g., residual heat from the violent stellar birth, glitches, and magnetic flux tube motion through the electron-proton sea of the core); (b) polar cap heating from an inflow of extremely relativistic $e^{-}$or $e^{+}$that have passed through an accelerator on the open field line bundle; (c) synchrotron radiation from $e^{ \pm}$pairs, created in the near magnetosphere above the stellar surface at altitude $\sim 3 R$; (d) inverse Compton scattering of radio photons by extremely relativistic $e^{-}$or $e^{+}$inflow or outflow on the open field lines of the pulsar. We shall consider here interpretation of X-ray observations from solitary (isolated) pulsars in terms of these assumed sources.

In $\S 2$ we summarize X-ray emission and pulsar properties: present data seem adequate for differentiating among possible sources. A dichotomy exists between the data from $\gamma$-ray pulsars and "ordinary" ones (those that are not observed to have strong $\gamma$-ray emission). The former show a power-law spectrum for their hard X-rays (1-10 keV) and no evidence for observation of the expected heated polar cap emission. The latter do not show the strong strong power-law emission, but heated polar cap emission does appear to have been observed. We interpret these differences as a consequence of $e^{ \pm}$production in the near magnetospheres of $\gamma$-ray pulsars but not in those of ordinary ones.

The locations of major particle accelerators in pulsar magnetospheres and the flow of particles through them may be different from those which power the much lower energy radio emission. We discuss relevant features of magnetospheric accelerators in $\S 3$.

Power-law emission in the keV range with the observed spectral extent, intensity, and angular distribution seem to be a natural consequence of the outer magnetospheric accelerator models, but not of polar-cap ones. Modelling the source of power-law emission is the subject of $\S 4$.

Copious $e^{ \pm}$production on closed field lines above the neutron star surface, no matter what its origin, would sustain a cyclotron-resonant X-ray reflecting mirror there (referred to as a "blanket"). This blanket would reflect X-rays from a hot polar cap in all directions except in a narrow cone through either of the two holes in the blanket where the open field line bundle penetrates through it. These cyclotron-resonant blankets are considered in $\S 5$. 
Such thick blankets should only exist on the closed field lines above the surfaces of $\gamma$-ray pulsars of which the hard X-ray power-law spectrum is from the synchrotron radiation of

$e^{ \pm}$pairs made in the near-magnetosphere. Direct observation of X-rays from heated polar caps through the holes of the open field line bundle would be expected to be rare: most of these X-rays from a hot polar cap would be reflected back toward the star by the blanket.

In $\S 6$ we discuss features of individual pulsars that need special consideration. The $\gamma$-ray pulsar Geminga has a soft X-ray $(0.1-1 \mathrm{keV})$ luminosity that is not as large as that from the heating of a polar cap expected from the down-flowing extremely relativistic $e^{-}$

or $e^{+}$that strike it. A possible mechanism for reducing the power of that inflow by inverse Compton scattering of inflowing extremely relativistic $e^{-}\left(e^{+}\right)$by radio photons emitted near the polar cap is presented.

The presence or absence of a strong blackbody component of hard X-rays depends on the formation of the blanket on closed field lines of the near magnetosphere. For most non- $\gamma$-ray pulsars, such blankets would not be expected, and direct observation of their hot polar caps is possible. In $\S 7$ we consider the effective areas of the X-ray emission and luminosities of hot polar caps.

If the blanket is present, soft X-rays $(0.1-1 \mathrm{keV})$ can escape from the star either by penetrating through the blanket itself, which gives a weakly modulated light curve, or by leaking out through the holes of the blanket on open field lines, which gives a strongly modulated one. The combination of these two sources might explain the pulsed fraction observed in various pulsars. Because of uncertainties with luminosity and density distribution, a quantitative calculation is not yet available. We only outline this concept in $\S 8$.

\section{X-ray Observations}

Following the detection by the Einstein satellite of several rotationally powered pulsars at X-ray wavelengths, their surface emission and magnetospheric processes have been studied using spectra and light curves obtained by the Röntgen Satellite (ROSAT) and the Advanced Satellite for Cosmology and Astrophysics (ASCA). About two dozen isolated pulsars have been detected by these instruments (Finley, Ögelman, \& Kızıloğlu 1992; Halpern \& Holt 1992; Ögelman \& Finley 1993; Yancopoulos, Hamilton, \& Helfand 1994; Manning \& Willmore 1994). X-ray spectra are usually fitted to either a thermal blackbody shape or a nonthermal power-law one.

Geminga is a radio-quiet pulsar with period $P=0.237 \mathrm{~s}$, surface magnetic field 
$B_{\mathrm{p}} \sim 1.6 \times 10^{12} \mathrm{G}$, and characteristic age $\tau=P / 2 \dot{P}=3.4 \times 10^{5}$ years Halpern \& Holt 1992; Bertsch et al. 1992). Halpern and Ruderman (1993) fitted the ROSAT PSPC spectrum of Geminga with a two-component model, either two blackbodies, or a blackbody plus a power-law. Both fits gave a satisfactory description of the spectrum. This ambiguity was resolved by combining ROSAT and $A S C A$ data: the bulk of the soft X-ray flux is a blackbody, and the hard X-ray spectrum is a power law (Halpern \& Wang 1997). The soft X-ray component is parameterized as a blackbody with $T=5.77_{-0.46}^{+0.37} \times 10^{5} \mathrm{~K}$ and a bolometric luminosity $1.47 \times 10^{31} \mathrm{ergs} \mathrm{s}^{-1}$; the hard X-ray spectrum is fitted by a power law with energy index $0.47_{-0.23}^{+0.25}$ and luminosity $8.13 \times 10^{29} \mathrm{ergs} \mathrm{s}^{-1}$ (Figure 1 ). The luminosities were calculated from the parallax distance of $160 \mathrm{pc}$ from the HST measurements (Caraveo et al. 1996).

PSR 1055-52 and PSR 0656+14 resemble Geminga in many respects: periods, loss rate of rotational energy, characteristic age, etc. (Table 1). They have also been fitted

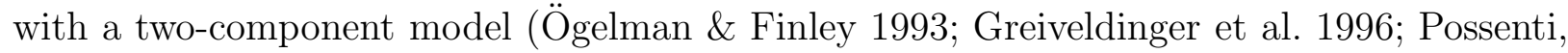
Mereghetti, \& Colpi 1996). The soft X-ray component has generally been interpreted as thermal emission from all or a part of the surface of the neutron stars. However, the source of the harder X-ray component has not been determined. Ögelman and Finley (1993) analyzed the ROSAT data from PSR 1055-52; their results were fitted to a blackbody together with a power law of energy index 0.4-0.5. Although Greiveldinger et al. (1996) favored a blackbody model to describe the hard X-ray component for a joint fit of $R O S A T$ and $A S C A$ data, our own reexamination of the same $A S C A$ data shows that a power law with energy index $0.5 \pm 0.3$ and luminosity $1.5 \times 10^{30} \mathrm{ergs} \mathrm{s}^{-1}$ (calculated at $d=500 \mathrm{pc}$ ) fits the spectrum equally well (Figure 2). PSR 1055-52 possesses a large pulsed fraction, 0.73

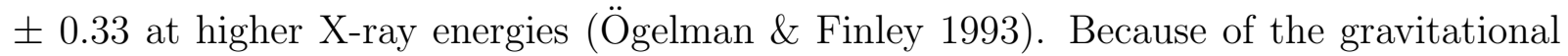
bending effect, pulsed fraction due to heated polar cap can rarely exceed $50 \%$, and a high pulsed fraction seems more easily compatible with a strongly beamed nonthermal hard X-ray component. [See simulations by Possenti et al. (1996) and Wang \& Halpern (1997).] The soft X-ray component has temperature $7.9_{-1.0}^{+0.6} \times 10^{5} \mathrm{~K}$ and bolometric luminosity $2.2 \times 10^{32} \mathrm{ergs} \mathrm{s}^{-1}$. Greiveldinger et al. (1996) also reported a joint analysis of ROSAT and $A S C A$ data for PSR $0656+14$. They required two blackbodies and a power law to fit the spectrum. Our own analysis verified the double blackbody model but failed to confirm the extra power-law component. The soft X-ray component has temperature $8.1_{-1.4}^{+0.5} \times 10^{5} \mathrm{~K}$ and bolometric luminosity $4.3 \times 10^{32} \mathrm{ergs} \mathrm{s}^{-1}$; the hard X-ray component has temperature $1.7_{-0.2}^{+0.2} \times 10^{6} \mathrm{~K}$ and a bolometric luminosity $2.6 \times 10^{31} \mathrm{ergs} \mathrm{s}^{-1}$. The luminosities are calculated at an estimated distance of $400 \mathrm{pc}$.

The older pulsars PSR 1929+10 and PSR 0950+08 have similar period, and roughly comparable characteristic ages, spin-down powers, and surface magnetic fields (Table 1). 
According to the cooling curves (Page \& Applegate 1992; Lattimer et al. 1991), the X-ray luminosity of a pulsar whose age exceeds $10^{6}$ years decreases sharply, and the typical energy of thermal photons from such an object is too low to create pairs in collisions with $\mathrm{GeV}$ photons. X-rays are detected from both pulsars by ROSAT (Yancopoulos et al. 1994; Manning \& Willmore 1994) and ASCA (Wang \& Halpern 1997). The spectra from both pulsars are fitted to a single blackbody spectrum from a small area. PSR $1929+10$ is best fitted with temperature $(5.14 \pm 0.53) \times 10^{6} \mathrm{~K}$ and a luminosity $1.54 \times 10^{30} \mathrm{ergs} \mathrm{s}^{-1}$ after adopting the distance $250 \mathrm{pc}$ of Yancopoulos et al. (1994). PSR 0950+09 has temperature $(5.70 \pm 0.63) \times 10^{6} \mathrm{~K}$ and a luminosity $4.67 \times 10^{29} \mathrm{ergs} \mathrm{s}^{-1}$, at the parallax distance of 125 pc (Gwinn et al. 1986).

At present, about twenty pulsars have been detected in soft X-rays, but few of them have sufficient photons for comprehensive spectral and temporal analysis. The above five objects, observed in the X-ray energy range $0.1-10.0 \mathrm{keV}$, represent pulsars of various classes. They all have periods of about $0.3 \mathrm{~s}$. Geminga, PSR 1055-52, and PSR 0656+14 have characteristic ages $\tau \sim 10^{5}$ years, whereas PSR 1929+10 and PSR 0950+08 have $\tau \sim 10^{7}$ years. Strong pulsed $\gamma$-ray emission is detected from Geminga (Bertsch et al. 1992) and PSR 1055-52 (Fierro et al. 1993), but such radiation is still uncertain for PSR 0656+14 (Ramanamurthy et al. 1996). While Geminga, PSR 1055-55, and PSR 0656+14 have typical dipole component magnetic fields $B_{\mathrm{p}} \gtrsim 10^{12} \mathrm{G}$, PSR $1929+10$ and PSR

$0950+08$ have weaker fields $\left(B_{\mathrm{p}}<10^{12} \mathrm{G}\right)$. Properties of these five pulsars are summarized in Table 1. Luminosities have a great uncertainty associated with the measurements of distance. We observe thermal soft X-ray emission from Geminga, PSR 1055-52, and PSR $0656+14$, and the emitting area is comparable to the entire surface of a neutron star. A thermal hard X-ray component is observed from PSR 1929+10, PSR 0950+08, and PSR 0656+14 from an area which occupies only a small fraction of the neutron star surface. A power-law spectrum in hard X-rays is observed from the $\gamma$-ray pulsars Geminga and PSR 1055-52. Light curves of the hard X-rays of both $\gamma$-ray pulsars have pulsed fractions exceeding $50 \%$, which we interpret as an indicator of nonthermal emission.

\section{Particle Flows in Pulsar Magnetospheres}

The Energetic Gamma Ray Experiment Telescope (EGRET) on the Compton Gamma Ray Observatory (CGRO) has detected six pulsars with strong $\gamma$-ray emission above 100 MeV: Crab, Vela, Geminga, PSR 1706-44, PSR 1055-52, and PSR 1951+32. Similarities among the $\gamma$-ray spectra and most of the light curves suggest that a similar powerful accelerator is operating in each of their magnetospheres. 
Models for magnetospheric accelerator gaps fall into two main classes. For a polar-cap accelerator (Ruderman \& Sutherland 1975, RS hereafter; Daugherty \& Harding 1982), generally assumed to power the radio emission of a pulsar, the acceleration of primary particles takes place relatively near the surface of a neutron star. In the central dipole approximation for the stellar magnetic field, all the radiation and pair production resulting from primaries which have passed through such accelerators would be restricted to be within the open field-line bundle that links the polar cap to the light cylinder $\left(r_{\mathrm{LC}} \equiv c / \Omega\right.$, in which $\left.\Omega=2 \pi P^{-1}\right)$. Outer-magnetospheric accelerators have been proposed for the emission of the energetic photons from $\gamma$-ray pulsars (Cheng, Ho, \& Ruderman 1986a,b, hereafter CHRa, CHRb). These accelerators could achieve much higher potential drops along $\mathbf{B}$,

$$
\Delta V \sim \frac{\Omega^{2} B_{\mathrm{p}} R^{3}}{c^{2}} \sim 10^{14} \mathrm{~V}
$$

with $B_{\mathrm{p}}$ the surface dipole field, than can polar cap accelerators. Pair production within such accelerators (e.g. from accelerator produced $\mathrm{GeV} \gamma$-rays colliding with keV X-rays from the stellar surface or its neighborhood) will result in a net flow of primary $e^{-}\left(e^{+}\right)$out of the star being balanced by an inward flow of $e^{+}\left(e^{-}\right)$from the starward end of the accelerator. Because of extensive $e^{ \pm}$flows generated by both polar cap and outer-magnetospheric accelerators, it is difficult to see how both could survive on the same field lines: pairs from one would be expected to quench the other. However, a polar cap might be linked to both kinds of accelerator as long as the field lines through each are different [e.g. upward curving with a net positive (negative) charge near the light cylinder for one and downward curving negative (positive) for the other, or lines passing through the charge reversing surface $\hat{\Omega} \cdot \mathbf{B}=0$ for the one but not for the other, etc.].

Arguments for important outer-magnetospheric accelerators as well as polar cap ones in $\gamma$-ray pulsars but not in ordinary ones include the following: (a) apparently different death lines for the two families (Chen \& Ruderman 1993); for example ordinary pulsar radio emission appears to cease when polar cap accelerator $e^{ \pm}$production is no longer possible while $\gamma$-ray pulsar emission seems to be quenched when putative outer-magnetospheric accelerators would no longer be able to sustain the mechanism of collision between GeV $\gamma$-rays and keV X-rays; (Thus, Geminga and PSR 1055-52 could maintain outer-magnetospheric accelerators, but PSR 1929+10 and PSR 0950+08 could not; the former are $\gamma$-ray pulsars, while the latter are not.) (b) strong optical emission coincident with X-ray emission from the Crab pulsar which is difficult to explain unless its source is synchrotron radiation in the outer magnetosphere; (c) escape of $10 \mathrm{GeV} \gamma$-rays from PSR 1951+32 and PSR 1706-44 which seems more difficult to accomplish if these $\gamma$-rays originate in the neighborhood of strong polar cap fields; (d) the $\gamma$-ray luminosity of these $\gamma$-ray pulsars with $P \gtrsim 10^{-1} \mathrm{~s}^{-1}\left(L_{\gamma} \sim 10^{34} \mathrm{ergs} \mathrm{s}^{-1}\right)$. From an outer-magnetospheric 
accelerator, because almost the full potential drop $\Delta V \approx 10^{14} \mathrm{~V}$ will be radiated away by each electron in the accelerator, we indeed expect just such a luminosity,

$$
L_{\gamma} \sim \dot{N}_{0} e \Delta V \sim 10^{34} \operatorname{ergs~s}^{-1},
$$

for $\dot{N}_{0}$ the maximum current through the accelerator (i.e. the Goldreich-Julian current)

$$
\dot{N}_{0} \sim \frac{\Omega^{2} B_{\mathrm{p}} R^{3}}{e c} \sim 10^{32} \mathrm{~s}^{-1} .
$$

We assume that outer-magnetospheric accelerators - or at least their starward flow of extreme relativistic leptons - are present in $\gamma$-ray pulsars. [We note, however, that the outer-magnetospheric accelerator model of Romani (1996) and co-workers does not include any such symmetry between outward $e^{-}\left(e^{+}\right)$and inward $e^{+}\left(e^{-}\right)$flow.] In the next section, an interpretation of X-ray data from $\gamma$-ray pulsars provides more evidence favoring outer-magnetospheric accelerators as a source of extremely relativistic starward particle flow.

\section{X-rays from $\gamma$-ray Pulsars}

The $\gamma$-ray pulsars Geminga, PSR 1055-52, and PSR 1951+32 all have a power-law hard $\mathrm{X}$-ray component with energy index $\sim 0.5$. If synchrotron radiation in the magnetosphere is responsible for this emission, an outer-magnetospheric model can explain its slope and intensity. The polar-cap accelerator model, on the other hand, has difficulties accounting for this component, as we describe below.

\subsection{Spectra}

The energy loss rate $\dot{E}$ of extremely relativistic charged particles flowing along curved paths is

$$
\dot{E}=\dot{\gamma} m c^{2}=-\frac{2}{3} \frac{e^{2} c}{s^{2}} \gamma^{4},
$$

with $s$ the radius of curvature. For $\gamma$-ray pulsars powered by outer-magnetospheric accelerators, the number of particles flowing through an accelerator along the open field line bundle is limited by the Goldreich-Julian current. Although an outer-magnetospheric accelerator might sustain a potential difference along open field lines as high as $\Delta V \sim 10^{14}$ $\mathrm{V}$, radiation reaction limits the energy of primary particles $E_{\mathrm{p}}(\mathrm{CHRb})$

$$
\gamma_{1} \equiv \frac{E_{\mathrm{p}}}{m c^{2}} \leq\left(\frac{\mathbf{E} \cdot \hat{\mathbf{B}}}{e} s^{2}\right)^{1 / 4} \sim 3 \times 10^{7},
$$


For a dipole field, $s \cong(r c / \Omega)^{1 / 2}$, with $r$ the radial distance. When primary particles flowing starward from an outer-magnetospheric accelerator approach the polar cap, $\gamma$ satisfies

$$
\frac{1}{\gamma^{3}}-\frac{1}{\gamma_{1}^{3}} \sim \frac{2 \Omega e^{2}}{m c^{3}} \ln \left(\frac{r_{\mathrm{LC}}}{r}\right),
$$

with $r \cong c t$. If $r \ll r_{\mathrm{LC}}, \gamma$ is insensitive to $r$ and $\gamma_{1}$; it remains approximately constant at

$$
\gamma \sim\left(\frac{2 \Omega e^{2}}{m c^{3}} \ln \frac{r_{\mathrm{LC}}}{r}\right)^{-1 / 3} \sim 10^{7} .
$$

Each such $e^{-}\left(e^{+}\right)$produces curvature radiation with

$$
E_{\gamma} \sim \hbar \gamma^{3}\left(\frac{c}{s}\right) \sim 100 \mathrm{MeV} .
$$

The number of such $\gamma$-rays radiated during the traversal along $\mathbf{B}$ a distance equal to the local radius of curvature is

$$
N_{\gamma} \sim \frac{\dot{E} s}{E_{\gamma} c} \sim \frac{\gamma e^{2}}{\hbar c} \sim 10^{5} .
$$

Photons from curvature radiation are initially emitted almost tangent to the local B within an angle $\gamma^{-1}$; after they proceed a distance $l$, they make an angle $\phi$ with the magnetic field line,

$$
\phi \sim l / s .
$$

These $10^{2} \mathrm{MeV} \gamma$-ray photons are converted into $e^{ \pm}$pairs at a significant rate as soon as they cross magnetic field lines that satisfy (RS)

$$
\frac{E_{\gamma} \sin \phi}{2 m c^{2}} \frac{B_{12}}{2} \geq 1,
$$

The angle $\phi$ becomes the pitch angle of the $e^{ \pm}$pair trajectories. With an outermagnetospheric accelerator model for starward primary particle flow, most $100 \mathrm{MeV}$ photons will be converted on closed field lines at $B \sim 10^{10} \mathrm{G}$, and $\phi \sim 90^{\circ}$. Each electron and positron carries about half the parent $\gamma$-ray energy, and quickly loses its momentum perpendicular to the local magnetic field by synchrotron radiation at the rate

$$
\frac{1}{\tau_{\mathrm{s}}} \approx\left(\frac{\gamma_{\perp}}{\gamma_{\|}}\right)\left(\frac{e^{2}}{m c^{3}}\right) \omega_{B}^{2} \sim 10^{11} \mathrm{~s}^{-1},
$$

with

$$
\begin{gathered}
\gamma_{\perp} \approx \gamma \sin \phi, \\
\gamma_{\|}=\frac{\gamma}{\gamma_{\perp}} \approx \frac{1}{\sin \phi},
\end{gathered}
$$




$$
\omega_{B} \equiv \frac{e B}{m c} .
$$

The distance that a charged particle can propagate before losing most of its energy is $\sim 0.3$ cm (Figure 3).

The initial characteristic frequency of synchrotron radiation of these locally created $e^{ \pm}$ is

$$
\omega_{\mathrm{c}} \cong \gamma_{\perp}^{2} \gamma_{\|} \omega_{B} \approx \gamma^{2} \sin \phi \omega_{B}
$$

Their intensity spectrum is

$$
I_{\mathrm{s}}(\omega) \cong \frac{1}{2}\left(\frac{1}{\omega \omega_{B} \sin \phi}\right)^{1 / 2} m c^{2} .
$$

This power law spectrum with energy index 0.5 is valid from the minimum value of the local cyclotron energy where the pairs are produced, up to several MeV:

$$
E_{\min } \sim \frac{\hbar \omega_{B}}{\sin \phi} \sim 0.1 \mathrm{keV}
$$

and

$$
E_{\max } \sim\left(\frac{E_{\gamma}}{2 m c^{2}}\right)^{2} \sin \phi \hbar \omega_{B} \sim 5 \mathrm{MeV}
$$

Because $\gamma$ in equation (7) is insensitive to details of the initial accelerator, the energy index of the power law spectrum should generally be $\cong 0.5$. This value agrees with the ASCA spectra: both Geminga and PSR 1055-52 are observed to have that energy index. A power-law spectrum with energy index 0.5 should be expected up to the MeV range for all $\gamma$-ray pulsars. The lower cutoff energy $E_{\text {min }}$ depends on the local magnetic field in which the $\gamma$-ray is converted to $e^{ \pm}$pairs and the pitch angle $\phi$.

Both Geminga and PSR 1055-52 have been observed with the HST; Geminga has a visual magnitude $V=24.5$ (Bignami et al. 1996), whereas PSR 1055-52 has $m=24.88$ at $3400 \AA$ (Mignani, Caraveo, and Bignami 1997). If the soft X-ray spectrum is correctly described as a blackbody one, the visible flux from Geminga is too large to be the long wavelength tail of the X-ray spectrum (Halpern, Martin, \& Marshall 1996). And in contrast to the analysis by Mignani et al. (1997) of PSR 1055-52, we find the flux from a blackbody extrapolation of the X-rays to be $(2.0-3.1) \times 10^{-31} \mathrm{ergs}^{-2} \mathrm{sm}^{-1} \mathrm{~Hz}^{-1}$, according to the best-fitted blackbody temperature by Greiveldinger et al. (1996), much less than the observed flux $1.3 \times 10^{-30} \mathrm{ergs} \mathrm{cm}^{-2} \mathrm{~s}^{-1} \mathrm{~Hz}^{-1}$ at $3400 \AA$. While the extrapolated blackbody falls below the optical flux, the fitted X-ray power-law component would exceed it. This observation conforms to our model, since we expect the low-energy cutoff of the synchrotron spectrum to fall at about $0.1 \mathrm{keV}$. Below the cutoff energy the spectrum should rise (with 
increasing energy) at the canonical low-frequency synchrotron index $-\frac{1}{3}$. Within the uncertainty associated with the power law best-fit, we estimate the cutoff energy to be 20-500 eV. Improved X-ray, UV and optical observations are needed to refine the cutoff energy.

For a polar-cap accelerator, the crucial differences from the above are the pitch angle $\phi$ and number of $\gamma$-ray photons $N_{\gamma}$ a primary particle can make (to be discussed in $\S 4.2$ ). For $\gamma$-rays emitted above a polar-cap accelerator in a dipole field, $\phi$ can never become much greater than $(R \Omega / c)^{1 / 2}$, unless the emission radius greatly exceeds $R$, in which case $B$ becomes too weak for pair creation. If the emission radius is much greater than $R$, equation (11) will not be satisfied, because $\phi$ increases proportionatedly to $r^{1 / 2}$, while the magnetic field $B$ decreases as $r^{-3}$. Those $\gamma$-rays that are converted would have $\phi \lesssim 10^{-1}$, and $E_{\text {min }}$ will be more than an order of magnitude greater than the spectral limit of equation (18). A $1 \mathrm{keV}$ lower bound does not seem to agree with observations.

\subsection{Luminosities}

The luminosity of the hard X-ray component (in $A S C A$ ) from Geminga and PSR 1055-52 for an outer-magnetospheric accelerator model is

$$
L_{A S C A} \sim f \dot{N}_{0} N_{\gamma} \int_{\omega_{1}}^{\omega_{2}} I_{\mathrm{s}}(\omega) d \omega \sim 10^{30} \operatorname{ergs~s}^{-1}
$$

with $\hbar \omega_{1}=0.7 \mathrm{keV}$ and $\hbar \omega_{2}=5.0 \mathrm{keV}$, the $A S C A$ coverage. The factor $f$ in equation (20) is the fraction of the Goldreich-Julian current that flows back out through the accelerator to the polar cap. For an outer-magnetosphere accelerator centered on the null surface $(\hat{\Omega} \cdot \mathbf{B}=0)$ this fraction depends on details of the outer-magnetospheric accelerator model, the angle between the spin and the dipole moment, etc. It is typically near $\frac{1}{2}$ for $\gamma$-ray pulsars not too far from their death lines (CHRb). The luminosity of equation (20) is close to the observed values of $L_{2}$ in Table 1 for Geminga and PSR 1055-52.

For polar-cap models, only $\gamma$-rays emitted by primary particles within a stellar radius $R$ or so of the stellar surface can make $e^{ \pm}$pairs (same argument as $\S 4.1$ ). Thus $N_{\gamma}$ will be reduced from the value in equation (9) by of order $R / s$, and the luminosity of equation (20) should be reduced by this same factor. The simulations by Daugherty and Harding (1996) (Figure 3 in their paper) support our estimate. For $\gamma$-ray pulsars with $P \sim 10^{-1} \mathrm{~s}$ and a dipole magnetic field approximation, $R / s \lesssim 10^{-2}$, so that $L_{A S C A} \lesssim 10^{28} \mathrm{ergs} \mathrm{s}^{-1}$, not enough to account for what is observed. 


\subsection{Light Curves}

If X-rays are produced above a polar-cap accelerator, $e^{ \pm}$pairs have to be created before $B$ drops below $\sim 10^{11} \mathrm{G}$. Therefore the angular size of the cone of emitted synchrotron radiation in X-rays should be of order $(3 R \Omega / c)^{1 / 2}$. Our estimate is supported by the simulations by Daugherty \& Harding (1996): their Figure 3 indicates that pair production can no long be sustained if $r \gtrsim 3 R$. The angular width of the X-ray cone should then not much exceed the angular spread of the open field line bundle from which the emission takes place. Analyses of radio emission generally place "conal emission" at $r \sim 10 R$ and "core emission" at $r \sim R$ (Rankin 1990, and references therein). The synchrotron X-ray emission from these same field lines should then be observed coincident in phase with the radio emission and with a width even less than that of conal radio emission. Neither is the case for PSR 1055-52, while no radio emission is yet confirmed from Geminga.

\subsection{The Crab Pulsar}

The spectrum of the Crab pulsar in the ROSAT and $A S C A$ regime is dominated by the radiation in two subpulses. That radiation is almost certainly from the same region and particles which are the source of its high-energy (up to at least $1 \mathrm{GeV}$ ) $\gamma$-rays, but not pairs created on closed field lines in the near magnetosphere discussed above. For X-rays from the latter we should look at the radiation between these subpulses, which should also have a broader angular spread than that in the two subpulses. The spectrum of this inter-pulse radiation has been isolated by Mineo et al. (1997) (also see Massaro et al. 1998). They find a power law for it, of which energy index (smaller by 0.32 than that in the subpulses) is 0.5 , consistent with that for Geminga and PSR 1055-52. This radiation extends up to $200 \mathrm{keV}$.

\section{Absence of Observable Hot Polar Caps}

Relativistic inflowing particles radiate away much of their energy before reaching the polar cap. According to equation (6) the residual energy of the charged particles impacting the polar cap is

$$
E_{\mathrm{f}}=m c^{2}\left[\frac{2 \Omega e^{2}}{m c^{3}} \ln \left(\frac{r_{\mathrm{LC}}}{R}\right)\right]^{-1 / 3} \sim 5.5 \mathrm{ergs} .
$$

With constant bombardment of these particles at a rate of $10^{32} \mathrm{~s}^{-1}$ (the Goldreich-Julian current) the polar cap would radiate X-rays with luminosity

$$
L_{\mathrm{X}}=f E_{\mathrm{f}} \dot{N}_{0} \cong 2.1 \times 10^{32} \operatorname{ergs~s}^{-1} .
$$


However, no evidence for such a strongly heated polar cap is found in Geminga or PSR 1055-52. The total thermal X-ray luminosity of PSR 1055-52 is $2.3 \times 10^{32} \mathrm{ergs} \mathrm{s}^{-1}$, whereas for Geminga it is $1.5 \times 10^{31} \mathrm{ergs} \mathrm{s}^{-1}$. In both cases X-rays seem to be emitted from a large fraction of the surface of the neutron star. We attribute the absence of observed hot polar caps to the formation of a reflective $e^{ \pm}$"blanket" above the stellar surface.

The creation of $10^{37} e^{ \pm}$pairs per second which produce the power-law synchrotron radiation in $\S 4$ would take place mainly on closed field lines in which $B \sim 10^{10} \mathrm{G}$ (at $\left.r \sim\left(\frac{B_{\mathrm{p}}}{10^{10}}\right)^{1 / 3} R \sim 6 R\right)$. As we discuss later, production of $e^{ \pm}$pairs at such a rate would have significant impact on the observability of X-ray emission from the stellar surface. Because we expect a $\gamma$-ray pulsar with an outer-magnetospheric accelerator to produce this number of $e^{ \pm}$pairs, but not so for an ordinary pulsar, the X-ray spectrum is expected to be different for these two families.

In order for a blanket to be sustainable, the $e^{ \pm}$annihilation rate in it must not exceed the pair injection rate there. This annihilation rate for a local $n_{ \pm}$is

$$
r_{ \pm} \sim\left(n_{ \pm}\right)^{2} \sigma_{ \pm}\left(\frac{c}{v_{ \pm}}\right) v_{ \pm} \sim 10^{14}\left(n_{ \pm}\right)_{14}^{2} \mathrm{~cm}^{-3} \mathrm{~s}^{-1}
$$

in which $\sigma_{ \pm} \frac{c}{v_{ \pm}}$is the annihilation cross section for non-relativistic $e^{ \pm}$pairs with velocities $v_{ \pm}$. It corresponds to a total near magnetospheric annihilation rate

$$
R_{ \pm} \sim 10^{34}\left(n_{ \pm}\right)_{14}^{2} \mathrm{~s}^{-1}
$$

In comparison the $e^{ \pm}$production rate on the closed field lines from equations (3) and (9), $N_{\gamma} \dot{N}_{0} \sim 10^{37} \mathrm{~s}^{-1}$, could balance $R_{ \pm}$with $n_{ \pm} \sim 5 \times 10^{15} \mathrm{~cm}^{-3}$. When a blanket is formed the lifetime of an $e^{ \pm}$pair is limited by the pair annihilation rate to about one second when the pair density is $10^{14} \mathrm{~cm}^{-3}$. If no blanket were to form and positrons flowed without interruption to the stellar surface, their residence time in the near magnetosphere would be a thousand times less. In that case there would not be a sufficient pair density to surround the star almost everywhere with a good reflecting blanket.

There are five forces acting on these $e^{ \pm}$pairs:

(i) the gravitational force,

$$
\mathbf{F}_{\mathrm{g}}=-\frac{G M m}{r^{2}} \hat{\mathbf{r}}
$$

(ii) the centrifugal force,

$$
\mathbf{F}_{\mathrm{c}}=m \Omega^{2} \mathbf{r}
$$


(iii) an electric force, which may exist within the open field line bundle

$$
\mathbf{F}_{\mathrm{E}}=e(\mathbf{E} \cdot \hat{\mathbf{B}}) \hat{\mathbf{B}} .
$$

(iv) the Lorentz force which confines $e^{ \pm}$to the local magnetic field lines, and

(v) the radiation force of scattered X-rays originally emitted from the surface of the neutron star.

We specialize our numerical calculations below to PSR 1055-52, but the formulae are applicable to other pulsars. The strong magnetic field restricts particle motion across a field line, therefore we consider only the component of forces along the magnetic field. Because $\mathbf{E} \cdot \mathbf{B}=0$ on closed field lines, and centrifugal force is insignificant in the region of interest $[r \sim 2-3 R$, to be shown later in equation (35)], it is sufficient to consider merely gravity and radiation. Zhu and Ruderman (1997), in a study of the $e^{ \pm}$annihilation line from the Crab pulsar, found that a balance between these forces can result in a stable equilibrium, in which an optically thick accumulation of $e^{ \pm}$pairs is formed over the neutron star.

A blackbody spectrum for polar-cap emission from the surface is

$$
I_{\mathrm{b}}(\omega)=L_{\mathrm{X}} \frac{15}{\pi^{4}} \frac{\hbar^{4}}{(k T)^{4}} \frac{\omega^{3}}{\exp (\hbar \omega / k T)-1},
$$

with $L_{\mathrm{X}}$ the bolometric luminosity $\left(2.1 \times 10^{32} \mathrm{ergs} \mathrm{s}^{-1}\right)$, and $T_{2}$ the polar cap temperature which is estimated to be

$$
k T_{2} \sim\left(\frac{L_{\mathrm{X}}}{\pi R^{3} \frac{\Omega}{c} \sigma_{\mathrm{SB}}}\right)^{1 / 4} \sim 0.5 \mathrm{keV}
$$

in which $\sigma_{\mathrm{SB}}$ is the Stefan-Boltzmann constant. Here we assume the canonical polar cap size. Our purpose is only to estimate but not obtain a definite numerical result, and later we will find out that the radial position of the blanket $\left(r_{\mathrm{pk}}\right)$ is insensitive to temperature $\left[r_{\mathrm{pk}} \propto T^{-1 / 3}\right.$, see equation (35)].

The magnetic field for a dipole at the center of the neutron star is

$$
\mathbf{B}(r, \theta)=\frac{B_{\mathrm{p}}}{2}\left(\frac{R}{r}\right)^{3}\left(2 \cos \theta \hat{\mathbf{e}}_{\mathrm{r}}+\sin \theta \hat{\mathbf{e}}_{\theta}\right) .
$$

In a strong magnetic field the averaged $e^{-}-e^{+}$cross section for X-ray scattering is approximated by (Blandford \& Scharlemann 1976)

$$
\sigma=\sigma_{\mathrm{T}}(\hat{\epsilon} \cdot \hat{\mathbf{B}})^{2}+\frac{2 \pi^{2} e^{2}}{m c}|\hat{\epsilon} \times \hat{\mathbf{B}}|^{2} \delta\left(\omega_{B}-\omega\right),
$$


with $\hat{\epsilon}$ the photon polarization and $\sigma_{\mathrm{T}}$ the Thomson cross section. In the optically thin case, the radiation force averaged over photon polarization is

$$
\mathbf{F}_{\mathrm{r}}=\left(\frac{\pi^{2} e^{2}}{4 m c^{2}}\right)\left(\frac{1+7 \cos ^{2} \theta}{1+3 \cos ^{2} \theta} \frac{I_{\mathrm{b}}\left(\omega_{B}\right)}{r^{2}}\right) \hat{\mathbf{r}} .
$$

The angle-averaged radiation force is plotted in Figure 4a. For the estimated polar cap temperature $0.5 \mathrm{keV}$, the stable equilibrium point is $r=6.9 R$. Electrons and positrons produced below this point will be pushed out along their magnetic field line to this radius; those above it will be pulled in toward it by gravity. When the number density of electrons and positrons grows large enough, the $e^{ \pm}$plasma becomes optically thick to the X-ray photons from the surface and the radiation force per lepton of equation (32) decreases. To make an optically thick layer of $e^{ \pm}$pairs for the polar cap X-rays, the required minimum number density is

$$
n_{\mathrm{t}}(r, \theta) \cong \frac{3}{2} \frac{B_{\mathrm{p}}}{\pi^{2} e R}\left(\frac{R}{r}\right)^{4} \frac{\left(1+3 \cos ^{2} \theta\right)^{3 / 2}}{1+7 \cos ^{2} \theta} .
$$

A typical $n_{\mathrm{t}}$ at $r=2 R$ is $\sim 5 \times 10^{13} \mathrm{~cm}^{-3}$. The maximum pair density that can be supported by the X-ray radiation force is reached when the total radiation force from complete absorption equals the gravitational attraction. An optically thick $e^{ \pm}$blanket begins to develop inward from the stable equilibrium point $r=6.9 R$ but cannot grow to smaller radii than the unstable equilibrium point $r=1.3 R$. The radiation force for this optically thick example is plotted in Figure $4 \mathrm{~b}$ : between $1.3 R$ and $6.9 R$, the radiation force equals the gravitational force. Below the unstable equilibrium point $e^{ \pm}$pairs are pulled by gravity back to the surface of the neutron star. The maximum supportable density is achieved when all of the initial momenta of the resonance scattered X-ray photons support the $e^{ \pm}$plasma, then

$$
n_{\max }(r, \theta) \cong \frac{45}{4 \pi^{5}} \frac{1}{G M m R c}\left(\frac{\hbar e B_{*}}{m c k T}\right)^{4}\left(\frac{R}{r}\right)^{13} \frac{L_{\mathrm{X}}}{\exp \left[\frac{\hbar e B_{*}}{m c k T}\left(\frac{R}{r}\right)^{3}\right]-1}
$$

in which $B_{*}=\frac{B_{\mathrm{p}}}{2}\left(1+3 \cos ^{2} \theta\right)^{1 / 2}$. The number density $n_{\max }$ is plotted in Figure 5 ; its maximum occurs at

$$
r_{\mathrm{pk}} \cong 0.616 R\left(\frac{\hbar e B_{\mathrm{p}}}{m c k T}\right)^{1 / 3}
$$

If the local density of $e^{ \pm}$pairs is greater than the maximum supportable value, pairs will be pulled toward to the star by gravity, otherwise they will be repelled from the star by radiation. When a steady state is reached, the local number density will be near the maximum supportable value, but essentially zero elsewhere because it drops sharply after the resonant peak [equation (34)]. The above formulae are valid on closed field lines, 
as mentioned earlier. However, the open field bundle subtends only a small solid angle, $\pi(2 R \Omega / c) \sim 6 \times 10^{-3}$ ster, thus the star is well blanketed.

With $k T_{2} \cong 0.5 \mathrm{keV}$, the blanket has maximum number density $n_{\max } \cong 5 \times 10^{15} \mathrm{~cm}^{-3}$ at radius $r_{\mathrm{pk}} \cong 2 R$. This is almost three orders of magnitude greater than that required for an optically thick blanket. This optically thick blanket prevents direct observation of the polar cap from most directions, because the unblanketed part only occupies $\sim 10^{-3}$ the total area of the sphere of radius $2 R$. Most of the photons from the polar cap will be reflected back to the surface of the neutron star (Figure 6). This process transfers emitted polar cap X-ray energy to the entire surface of the neutron star, from which it is reradiated away at a lower temperature

$$
T_{1} \sim\left(\frac{L_{\mathrm{X}}}{4 \pi R^{2} \sigma_{\mathrm{SB}}}\right)^{1 / 4} \sim 7.4 \times 10^{5} \mathrm{~K} .
$$

This temperature is in reasonable agreement with the observation. The contribution of cyclotron resonant back-scattering onto the stellar surface gives rise to thermal radiation from the surface which is in addition to that from the cooling of the neutron star which was presumably very hot when it was formed; it might explain the huge difference of soft X-ray luminosities for Geminga and PSR 1055-52 discussed below. The cooler surface radiation will have to pass through its own blanket at $r \sim 5 R$ or through the open field line "holes" where $e^{ \pm}$blankets can be kept from forming by the strong relativistic current flows. We discuss the escape of soft X-rays further in $\S 8$.

\section{Resonant Inverse Compton Scattering on Radio Photons}

If the true ages of Geminga and PSR 1055-52 are near their characteristic ages, it is difficult for a cooling model to explain their enormous difference in luminosity since they have similar characteristic ages, and Geminga is younger. One possible explanation is illumination of the extremely relativistic inflowing particles by even a tiny fraction of the radio emission which is generally assumed to be generated by a polar cap accelerator. Although no such radio-frequency radiation has been observed from Geminga, it is not implausible that this is because we do not intercept its emission beam. Special near-surface magnetic field configuration (especially for core radio beam emission) or gravitational bending of $\gamma$-rays might allow interception of a small part of the radio beam far from the stellar surface by the postulated extreme relativistic inflow of particles directed down toward the polar cap. Then cyclotron resonant inverse Compton scattering between these relativistic $e^{-}\left(e^{+}\right)$and the radio beam may become important. In the rest frame of the inflowing particles, the radio frequency is upshifted by $\gamma\left(\omega^{\prime} \approx \gamma \omega\right)$. For a radio photon of 
frequency $500 \mathrm{MHz}$ and $\gamma \sim 10^{7}$, there is a resonance at $B \approx 10^{9} \mathrm{G}$ or $r \approx 10 R$. When inverse Compton scattering occurs, the final scattered photon energy is

$$
E_{\gamma} \sim \gamma^{2} \hbar \omega .
$$

For $\gamma \sim 10^{7}$, and frequency of radio photons $\sim 500 \mathrm{MHz}$, the final energy of these scattered photons is $\sim 100 \mathrm{MeV}$, and they will be converted to $e^{ \pm}$pairs before approaching the surface. The $\gamma$-ray energy will thus be converted into the synchrotron radiation as discussed in $\S 4$.

With the resonant cross section $\sigma_{\mathrm{r}} \cong \frac{2 \pi^{2} e^{2}}{m c} \delta\left(\omega_{B}-\omega\right)$, and a Goldreich-Julian number density for inflowing primaries, the optical depth for the radio photons passing through the inflowing particles is

$$
\tau_{\mathrm{i}}=\int \sigma_{\mathrm{r}} n d r \cong \frac{\pi}{3} \frac{r_{\mathrm{i}} \Omega}{c} \cong 10^{-2},
$$

with $r_{\mathrm{i}}$ the resonance radius $\sim 10 R$. Hence only a very small fraction of any incident number of radio photons would be scattered. [Because the fraction of radio photons interacting with leptons is negligible, inverse Compton scattering cannot be responsible for the absence of radio emission from Geminga.] The energy loss rate of charged particles to resonant inverse Compton scattering would then be

$$
\dot{E}_{\mathrm{e}} \sim \tau_{\mathrm{i}} \cdot \frac{f_{\mathrm{r}} L_{\text {radio }}}{\hbar \omega} \cdot \gamma^{2} \hbar \omega \sim 10^{38} \mathrm{f}_{\mathrm{r}} \mathrm{erg} \mathrm{s}^{-1},
$$

with $f_{\mathrm{r}}$ the small fraction of the radio beam that encounters relativistic particles and $L_{\text {radio }} \sim 10^{25} \mathrm{ergs} \mathrm{s}^{-1}$ the radio luminosity. Although this estimate involves many uncertainties, it shows that resonant inverse Compton scattering can be extremely efficient in transferring energy from particles into $\gamma$-rays: a very minuscule fraction $f_{\mathrm{r}} \gtrsim 10^{-6}$ could take away most of the energy of the primary inflowing particles and reduce polar cap heating. Such a mechanism would not necessarily be a universal one and there is no obvious reason that polar-cap inflow in PSR 1055-52 must be similarly affected.

\section{Heated Polar Cap Areas}

The backflow of extremely relativistic particles heats the polar cap region. The predicted polar cap heating varies among models (Cheng \& Ruderman 1980; Arons 1981a; Halpern \& Ruderman 1993). The residual energy $E_{\mathrm{f}}$ of each relativistic particle is about 5 ergs [equation (21)] from an outer-magnetospheric accelerator whose inflow is uninterrupted (i.e., not the case proposed for Geminga in §6), whereas $E_{\mathrm{f}} \sim e \Delta V \sim 1 \mathrm{erg}$ for a polar-cap accelerator. An outer-magnetospheric accelerator can be a copious supplier of charged 
particles; nearly the full Goldreich-Julian current would be expected to pass through it. The full Goldreich-Julian current might not be achieved for backflow through a polar cap accelerator and we can estimate only an upper limit for the heated polar-cap luminosity in that case (Arons 1981b). As discussed in $\S 5$, a polar cap heated by an outer-magnetospheric accelerator is not expected to be directly visible unless an observer looks in along the dipole axis. We consider below only the observable polar cap heating of ordinary pulsars. The three pulsars in Table 1 with thermal emission from a tiny area are prospective candidates for such polar cap heating. However, their temperatures and luminosities vary greatly. While PSR $1929+10$ and PSR $0950+08$ have $\sim 10^{-2}$ smaller polar cap areas than that of central dipole models, PSR 0656+14 has a 30 times larger one (Table 1). We emphasize that the fitted luminosity and area depend on the assumed viewing geometry and distance, as well as on possibly important atmosphere effects (e.g. Meyer, Pavlov, \& Mészáros 1994).

The total magnetic flux $\Phi$ out of a polar cap is determined by the observed pulsar spin-down power $(I \Omega \dot{\Omega})$ :

$$
A_{\mathrm{pc}} B_{\mathrm{pc}} \equiv \Phi \sim\left(\frac{c I \dot{\Omega}}{\Omega}\right)^{1 / 2} .
$$

For a central dipole model the polar cap area

$$
A_{\mathrm{pc}}=A_{0} \equiv \pi R^{3} \frac{\Omega}{c}
$$

and

$$
B_{\mathrm{pc}}=B_{\mathrm{p}}
$$

An anomalously large (small) polar cap area $A_{\mathrm{pc}}$, relative to $A_{0}$, corresponds to a polar cap $B_{\mathrm{pc}}$ which is much smaller (larger) than $B_{\mathrm{p}}$. While a central dipole model gives a good description for the magnetic field far from the star, it may be inadequate for the surface field. For example, an off-center dipole, special evolutionary features not describable by a simple dipole, or effects of the strong diamagnetism of the superconducting core of the star may all lead to a $B_{\mathrm{pc}}$ very different from $B_{\mathrm{p}}$.

We consider below two possible consequences for $B_{\mathrm{pc}}$ and $B_{\mathrm{p}}$ which follow from an evolutionary model for the surface field of spin-down pulsars.

\subsection{PSR 0656+14}

PSR 0656+14 shares many similarities with Geminga and PSR 1055-52, but detection of $\gamma$-ray emission from it remains weak and inconclusive (Ramanamurthy et al. 1996). 
PSR 0656+14 lies very close to the $\gamma$-ray death line, which makes it difficult to decide theoretically if it should be a $\gamma$-ray pulsar. According to the model in $\S 5$, there is an important connection between strong $\gamma$-ray emission and the nature of the hard X-ray component in the sense that a power law of energy index 0.5 is to be associated with a $\gamma$-ray pulsar. In addition, an $e^{ \pm}$resonance blanket is produced by a $\gamma$-ray pulsar, which should prevent us from observing the heated polar cap directly. Since the X-ray spectrum of PSR $0656+14$ can be fitted with a double blackbody model (Table 1), we infer that it should not be a $\gamma$-ray pulsar, or at least not one with the magnitude of particle inflow of an outer-magnetospheric accelerator. From the observed temperature and luminosity of the hotter component, the polar cap size appears to be about 30 times greater than the canonical value $A_{0}$. A polar cap with lower temperature and larger area implies a locally much weaker magnetic field than that of a central dipole. We attribute this difference to the circumstance that PSR 0656+14 is a nearly aligned pulsar (Lyne \& Manchester 1988).

The interior of a neutron star consists of superfluid neutrons, and superconducting protons. A spinning neutron star with an angular velocity $\Omega$ has an array of quantized vortex lines parallel to its spin axis. Any magnetic field passing through the superconducting protons of the core is expected to be organized into quantized flux tubes below the stellar crust. In contrast to the quasi-parallel neutron vortex line array, the flux tube array is expected to have a complicated twisted toroidal and poloidal structure to achieve magnetohydrodynamic stability in the deep interior of a conducting star (Flowers \& Ruderman 1977). The vortex array of a spinning-down neutron star must expand with stellar spin-down. Because the core of a neutron vortex and a flux tube interact strongly as they pass through each other, the moving vortices push or pull on the flux tube array. This interaction results in flux tubes either moving together with the neutron vortices, or being cut through if the flux tube array cannot respond fast enough. The superfluid vortices move outward with a velocity

$$
v_{\mathrm{V}}=r_{\perp} \frac{\dot{P}}{2 P},
$$

with $r_{\perp}$ the distance from the vortex to the spin axis. Near the spin axis, the vortices move slowly; away from it, the vortices move much faster. If we assume that a pulsar was created with an almost axisymmetric magnetic field on its surface, and the axis of magnetic dipole happens to be almost aligned with the spin axis (the apparent case for PSR 0656+14), the fast moving outer vortices cut through the magnetic flux tubes, while much more slowly moving inner vortices drag the magnetic flux tubes with them and leave behind a region with a much weaker magnetic field around the spin axis (Figure 7). The last closed field lines become very distorted near the surface, and the actual polar cap will be larger than that of a pure central dipole field. [According to the model of Arons and co-workers (Scharlemann, Arons, \& Fawley 1978), polar cap accelerators form only on "favorably" 
curved field lines, those bend toward the axis of rotation. An aligned pulsar with a central dipole field would have no favorably curved lines, unlike the configuration of Figure 7, which would give rise to strong $e^{ \pm}$production above the polar cap.] If the pulsar were born with period $P_{\mathrm{i}} \sim 20 \mathrm{~ms}$, the present magnetic field near the spin axis should be diminished by a factor $P / P_{\mathrm{i}} \sim 20$, and the region close to the spin axis should have a weak magnetic field $B_{\mathrm{pc}} \sim 10^{11} \mathrm{G}$. The expected polar cap temperature should accordingly be lower than in a central dipole model:

$$
T \sim\left(E_{\mathrm{f}} \frac{\Omega B_{\mathrm{pc}}}{2 \pi e} \frac{1}{\sigma_{\mathrm{SB}}}\right)^{1 / 4} \sim 2 \times 10^{6} \mathrm{~K}
$$

We emphasize that it is only when an important platelet, one that contains the polar cap, happens to be pierced by the spin-axis that it can be pulled apart in this way during spin down. This is an exception to the usual case in which the platelets remain intact $(\S 7.2)$. We also note that for this special geometry the total dipole field is least affected by the spin-down history of the star. The original field has been moved away from the spin axis but has not been pushed out of the core of the star. [Despite its age $\tau \sim 1.1 \times 10^{5}$ years, PSR $0656+14$ has an average surface dipole field $\approx 4.7 \times 10^{12} \mathrm{G}$, very close to that of a young pulsar.] It is only after such expulsion has occurred that reconnection can begin to diminish greatly the dipole moment of the spinning pulsar (Ruderman, Zhu, \& Chen 1998).

\subsection{PSR $1929+10$ and PSR $0950+08$}

PSR 1929+10 and PSR 0950+08 are two relatively old pulsars. Their surfaces are expected to have cooled to less than $10^{5} \mathrm{~K}$ (Nomoto \& Tsuruta 1987; Lattimer et al. 1991; Page \& Applegate 1992). Therefore, the X-rays from these pulsars detected by ROSAT and $A S C A$ and fitted by temperatures $>1 \times 10^{6} \mathrm{~K}$, cannot be coming from the cooling surface but rather from small areas that are reasonably associated with polar cap heating.

Because of their small spin-down power $\left(\sim 10^{33} \mathrm{ergs} \mathrm{s}^{-1}\right)$, both pulsars are probably unable to sustain outer-magnetospheric accelerators (e.g. Chen \& Ruderman 1993). Both pulsars have rather weak surface dipole magnetic fields, $B_{\mathrm{p}} \approx(2-5) \times 10^{11} \mathrm{G}$. This might be an indication of an evolution of the magnetic field from an initially stronger one. As a young pulsar spins down, the initial hot polar caps move with the highly conducting crust in which they are embedded. When $P$ reaches a critical value $P_{\mathrm{c}}$, substantial cracking of cool crust begins. In the subsequent spin-down, when $P>P_{\mathrm{c}}$, the average surface dipole field $\langle B(P)\rangle$ decreases inversely with $P$ because the movement of the core superfluid neutron vortex array pulls core flux and the crust through which it penetrates (Ruderman 1991; 
Chen, Ruderman, \& Zhu 1998), so

$$
\langle B(P)\rangle=\left\langle B\left(P_{\mathrm{c}}\right)\right\rangle P_{\mathrm{c}} / P .
$$

Relatively strong uniform platelet fields $\left(B_{\mathrm{pl}}\right)$ remain frozen at

$$
B_{\mathrm{pl}}=\left\langle B\left(P_{\mathrm{c}}\right)\right\rangle \sim B(\text { Vela }) \sim 3 \times 10^{12} \mathrm{G} .
$$

these platelets move away from each other during the spin-down era but the stress within them is less able to break them apart (except in the special case of §7.1). The more dilute surface flux is then concentrated in small areas. The area of a polar cap is

$$
A_{\mathrm{pc}} \sim \frac{\langle B(P)\rangle}{B_{\mathrm{pl}}} \cdot A_{0} \sim \frac{1}{10} A_{0} .
$$

The temperature of the heated part of the polar cap is

$$
T \sim\left(E_{\mathrm{f}} \frac{\Omega B_{\mathrm{pl}}}{2 \pi e} \frac{1}{\sigma_{\mathrm{SB}}}\right)^{1 / 4} \sim 5 \times 10^{6} \mathrm{~K} .
$$

This result agrees with observation, $T \sim 5 \times 10^{6} \mathrm{~K}$ for PSR $1929+10$ and $T \sim 6 \times 10^{6} \mathrm{~K}$ for PSR 0950+08.

\section{Soft X-ray Modulation}

Three of the pulsars discussed in this paper are detected in soft X-rays, presumably thermal emission from the entire surface of the neutron stars. The emission is highly modulated, with a typical pulsed fraction of $20 \%$. This feature implies an anisotropic structure on or near the surface of a neutron star. The blanket model of $\S 5$ gives such an anisotropy. For the $\gamma$-ray pulsars Geminga and PSR 1055-52, copious $e^{ \pm}$pair production on closed field lines is a plausible consequence of strong $\gamma$-ray production. PSR $0656+14$, which has a weak field polar cap region surrounded by a stronger field closed field line region (§7.1), should have copious $e^{ \pm}$pair production on closed field lines surrounding its polar cap accelerator. A cyclotron resonant blanket could form around the hot polar cap. Thus, we expect an optically thick surrounding blanket above all these three neutron stars. X-ray photons will escape blanketed neutron stars either through the two holes of the blanket on their open field lines, or by diffusing through the blanket itself. The ratio of these two fluxes depends on the size of the holes relative to the area of the blanket, and the probability of penetrating through the blanket. If a photon falls on the hole, the probability of penetration is one; if a photon hits the blanket, the probability of penetration 
is proportional to $\left(1+\tau_{\mathrm{b}}\right)^{-1}$ in which $\tau_{\mathrm{b}}(\omega)$ is the frequency-dependent optical depth. [This should include both diffusion through the blanket and the special incident angles and polarizations for which $\hat{\epsilon} \times \hat{\mathbf{B}}=0$ is a good approximation in equation (31).] Then fraction the flux through the holes $\left(f_{\mathrm{h}}\right)$ versus that through the blanket $\left(f_{\mathrm{b}}\right)$ is

$$
\frac{f_{\mathrm{h}}}{f_{\mathrm{b}}}=\frac{2 \pi r^{3} \frac{\Omega}{c}}{4 \pi r^{2}}\left(\tau_{\mathrm{b}}+1\right) .
$$

The blanket area is roughly $10^{3}$ times greater than the hole size, and the expected optical depth $\tau_{\mathrm{b}}$ is roughly 10-200, depending on the photon frequency, incident angle, and polarization. As the star spins the photons escaping from the blanket should be more uniformly distributed than those from the holes. Then the strongly modulated portion of the light curve of soft X-rays could be from hole emission and the relatively non-varying part from passage through the blanket.

In fitting theoretical spectra to observed ones it should be emphasized that the areas and attitudes of both the open field holes in the blanket through which "hohlraum" radiation is mainly emitted and the blanket transmission $\left(1+\tau_{\mathrm{b}}\right)^{-1}$ are energy dependent. When $f_{\mathrm{b}} \ll f_{\mathrm{h}}$ and $r \gg\left(c R^{2} / \Omega\right)^{1 / 3}$, the emitted radiation resembles that from a classical "hohlraum" at the temperature of the stellar surface. Radiation is almost all reflected by the blanket, and absorbed and reemitted by the stellar surface before it escapes through the two small open field line holes. X-rays emerging from the two open field line holes would not be observed to have the canonical blackbody spectrum because the "hohlraum" radiation would have an emitting area which depends on the photon energy $E$,

$$
A_{\mathrm{h}}=\left(\frac{\pi R^{3} \Omega}{c}\right)\left(\frac{\hbar e B_{\mathrm{p}}}{m c E}\right) .
$$

This energy-dependent area is independent of the surface field structure when local $B \ll B_{\mathrm{p}}$, so $A_{\mathrm{h}}$ is not an adjustable parameter. However, quantitative comparisons of observations and models are not yet available because of uncertainties associated with luminosity measurements and the density distribution of blanket $e^{ \pm}$pairs needed to determine $\tau_{\mathrm{b}}$.

\section{Conclusions and Future Observations}

We have argued that if the $\mathrm{keV} \mathrm{X}$-ray emission from $\gamma$-ray pulsars is indeed synchrotron radiation with an energy index 0.5 , then outer-magnetospheric accelerators are consistent with the energy range over which this power law is observed, the X-ray luminosity in the observed $0.7-5.0 \mathrm{keV}$ range, and the broad modulated light curve. We find it difficult to see how polar-cap accelerator models for this synchrotron radiation can give these same results. 
If the expected $10^{37} \mathrm{~s}^{-1} e^{ \pm}$pairs are produced on the closed field lines above the stellar surface of $\gamma$-ray pulsars, cyclotron resonant blankets are formed which greatly obscure $\mathrm{X}$-rays emitted from that surface. Observations are not inconsistent with what is expected in the presence of such blankets.

Our model implies that a $\gamma$-ray pulsar should have a power-law spectrum of energy index 0.5 in X-rays extending from $0.1 \mathrm{keV}$ to a few $\mathrm{MeV}$, but polar cap heating will not usually be directly observable. This can be tested with more sensitive higher-energy X-ray data on the pulsars discussed in this paper, as well as others. These data can be obtained with longer exposures by $A S C A$, or by the next generation of X-ray missions such as $A X A F$, $X M M$, and $A S T R O-E$. The validity of the power-law interpretation of the hard X-ray flux should also be tested by observing its extension to a few $\mathrm{MeV}$, and its low-energy cutoff at $\sim 0.1 \mathrm{keV}$ with improved soft X-ray, UV, and optical spectra. Currently ROSAT data show that, in addition to the $\gamma$-ray pulsars listed in Table 1, PSR 1951+32 also has a power-law spectrum of energy index $0.6_{-0.2}^{+0.2}$ (Safi-Harb, Ögelman, \& Finley 1995); this result is an encouraging compliment to the model. Additional data from $A S C A$ should be extremely relevant.

For $\gamma$-ray pulsars, creation of $10^{37} \mathrm{~s}^{-1} e^{ \pm}$pairs is expected. There would be an expected $e^{+}+e^{-} \rightarrow \gamma+\gamma$ slightly red-shifted annihilation line of twice this strength. In the case of the Crab pulsar and possibly some other $\gamma$-ray pulsars, mechanisms exist which might raise this estimate by $10-10^{2}$ [e.g. some of the few $\mathrm{MeV} \gamma$-rays become converted at $B \sim 10^{12}$ G, see Zhu and Ruderman (1997)]. A line this weak is not detectable (e.g. by OSSE) at typical $\gamma$-ray pulsar distances. It might be observed if a $\gamma$-ray pulsar is someday found at a distance $\sim 100 \mathrm{pc}$ or less.

There are about 30 Galactic $\gamma$-ray sources detected by EGRET. Except for the few identified as pulsars, the nature of most of them remains uncertain. The discovery of Geminga as a radio-quiet pulsar opened up the possibility that additional pulsars might be detected in $\gamma$-rays but not in radio. If $\gamma$-rays are produced in the outer-magnetospheric accelerator, they will have a large latitudinal coverage. Because radio emission is generally believed to be produced on the open field line bundle near the polar cap, it has a narrow beam. In this picture, there is a large probability that the radio beam does not intersect the Earth while the $\gamma$-ray beam does. Thus, the population of radio-quiet $\gamma$-ray pulsars is potentially high enough to account for the majority of the unidentified Galactic EGRET sources (Halpern \& Ruderman 1993; Yadigaroglu \& Romani 1995). It is difficult to discover periodicity in the $\gamma$-ray data because of the paucity of photons, although this may be possible with future $\gamma$-ray missions such as GLAST. The identification of Geminga was made from its X-rays, of which the count rate is relatively high. X-ray counterparts of 
high-energy $\gamma$-ray sources may be more generally useful in finding Geminga-like pulsars. The relative abundance of X-ray photons allows spectral analysis, and our model predicts that a power-law spectrum in hard X-rays is associated with a $\gamma$-ray pulsar. This signature might provide important constraints in selecting the prospective pulsar candidates.

We thank K. Chen, J. Arons and E. Massaro for enlightening discussions. FYHW acknowledges support from NASA grant NAG 5-2524, JPH from NASA grant NAG 5-3229, MR and TZ from NASA grant NAG 5-2841. We also thank our referee for his comments. This paper is contribution 643 of the Columbia Astrophysics Laboratory. 


\section{REFERENCES}

Arons, J. 1981a, ApJ, 248, 1099

Arons, J. 1981b, in IAU Symp. 95, Pulsars, eds. W. Sieber \& R. Wielebinski (Dordrecht: Reidel), 69

Bertsch, D. L., et al. 1992, Nature, 357, 306

Bignami, G. F., Caraveo, P. A., Mignani, R., Edelstein, J., \& Bowyer, S. 1996, ApJ, 456, L111

Blandford, R. D., \& Scharlemann, E. T. 1976, MNRAS, 174, 59

Caraveo, P. A., Bignami, G. F., Mignani, R., \& Taff, L. G. 1996, ApJ, 461, L91

Chen, K., \& Ruderman, M. 1993, ApJ, 402, 264

Chen, K., Ruderman, M., \& Zhu, T. 1998, ApJ, in press

Cheng, A. F., \& Ruderman, M. A. 1980, ApJ, 235, 576

Cheng, K. S., Ho, C., \& Ruderman, M. 1986a, ApJ, 300, 500 (CHRa)

Cheng, K. S., Ho, C., \& Ruderman, M. 1986b, ApJ, 300, 522 (CHRb)

Daugherty, J., \& Harding, A. K. 1982, ApJ, 252, 337

Daugherty, J., \& Harding, A. K. 1996, ApJ, 458, 278

Fichtel, C. E., et al. 1975, ApJ, 198, 163

Fierro, J. M., et al. 1993, ApJ, 413, L27

Finley, J. P., Ögelman, H., \& Kızıloğlu, Ü. 1992, ApJ, 394, L21

Flowers, E., \& Ruderman, M. A. 1977, ApJ, 215, 302

Greiveldinger, C. et al. 1996, ApJ, 465, L35

Gwinn, C. R., Taylor, J. H., Weisberg, J. M., \& Rawley, L. A. 1986, AJ, 91, 338

Halpern, J. P., \& Holt, S. S. 1992, Nature, 357, 222

Halpern, J. P., Martin, C., \& Marshall, H. L. 1996 ApJ, 473, L37

Halpern, J. P., \& Ruderman, M. 1993 ApJ, 415, 286 
Halpern, J. P., \& Wang, F. Y.-H. 1997, ApJ, 477, 905

Lattimer, J. M., Pethick, C., Prakash, C. J., \& Haensel, P. 1991, Phys. Rev. Lett., 49, 1373

Lyne, A. G., \& Manchester, R. N. 1988, MNRAS, 234, 477

Manning, R. A. \& Willmore, A. P. MNRAS, 266, 635

Massaro, E., Litterio, M., Cusumano, G., \& Mineo, T. 1998, in Proc. Conf. "The Active X-ray Sky", eds. L. Scarsi et al. (in press)

Meyer, R. D., Pavlov, G. G., \& Mészáros, P. 1994, ApJ, 433, 265

Mignani, R., Caraveo, P. A., \& Bignami, G. F. 1997, ApJ, 474, L51

Mineo, T., Cusumano, G., Segreto, A., Massaro, E., Dal Fiume, D., Giarrusso, S., Matteuzzi, A., Nicastro, L., \& Parmar, A. N. 1997, A\&A, 327, L21

Nomoto, K., \& Tsuruta, S. 1987, ApJ, 312, 711

Ögelman, H., \& Finley, J. P. 1993, ApJ, 413, L31

Page, D., \& Applegate, J. H. 1992, ApJ, 394, L17

Possenti, A., Mereghetti, S., \& Colpi, M. 1996, A\&A, 313, 565

Ramanamurthy, P. V., Fichtel, C. E., Kniffen, D. A., Sreekumar, P., \& Thompson, D. J. 1996, ApJ, 458, 755

Rankin, J. M. 1990, ApJ, 352, 247

Romani, R. W. 1996, ApJ, 470, 469

Ruderman, M. A., \& Sutherland, P. G. 1975, ApJ, 196, 51 (RS)

Ruderman, M. 1991, ApJ, 382, 587

Ruderman, M., Zhu, T., \& Chen, K. 1998, ApJ, in press

Safi-Harb, S., Ögelman, H., \& Finley, J. P. 1995, ApJ, 439, 722

Scharlemann, E. T., Arons, J., \& Fawley, W. M. 1978, ApJ, 222, 297

Thompson, D. J., Fichtel, C. E., Hartman, P. C., Kniffen, D. A., \& Lamb, R. C. 1977, ApJ, 213,252 
Wang, F. Y.-H., \& Halpern, J. P. 1997, ApJ, 482, L159

Yadigaroglu, I.-A., \& Romani, R. W. 1995, ApJ, 449, 211

Yancopoulos, S., Hamilton, T. T., \& Helfand, D. J. 1994, ApJ, 429, 832

Zhu, T., \& Ruderman, M. 1997, ApJ, 478, 701 


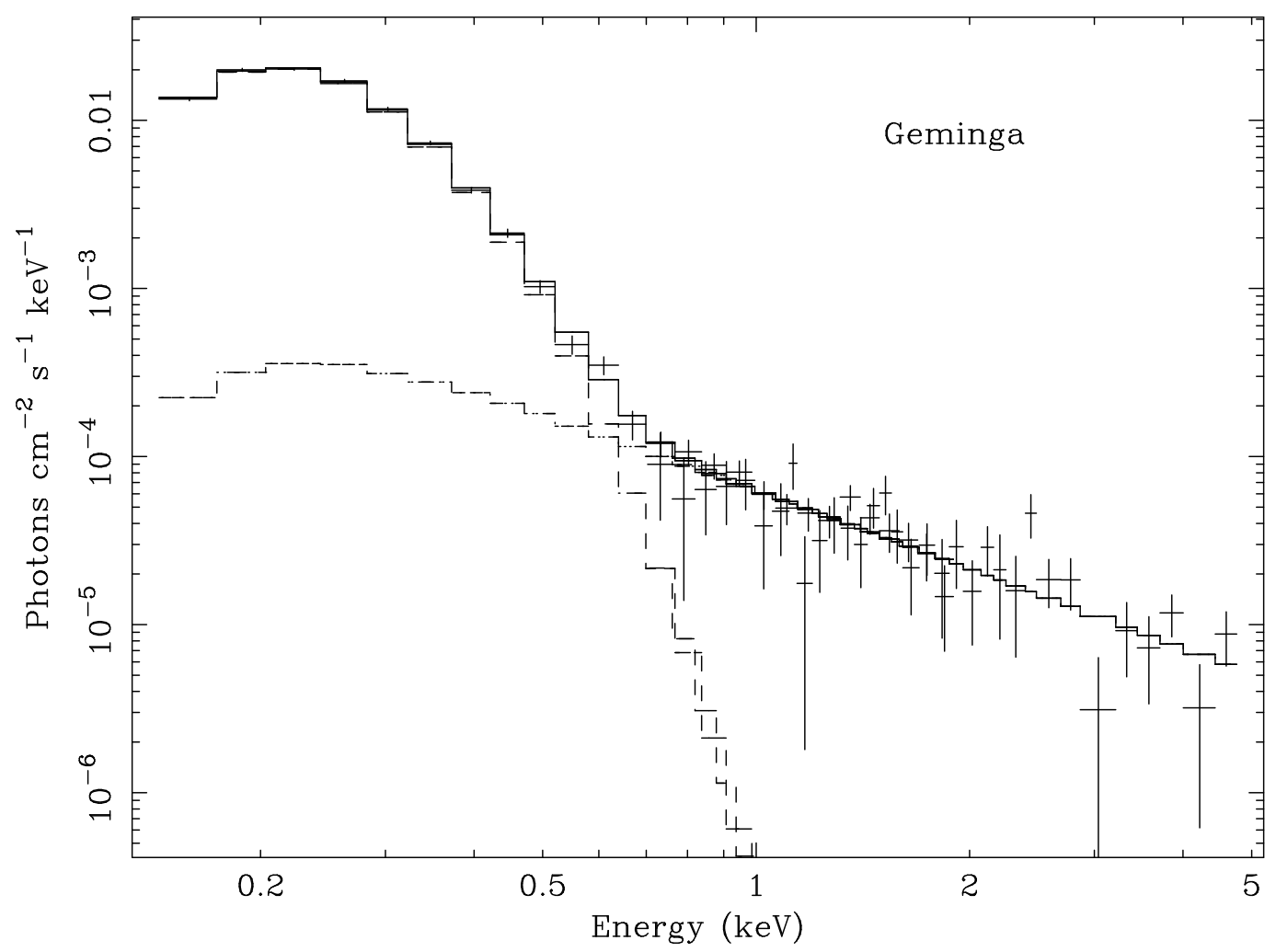

Fig. 1.- Fit of the blackbody plus power-law model to the $A S C A$ and ROSAT spectra of Geminga. 


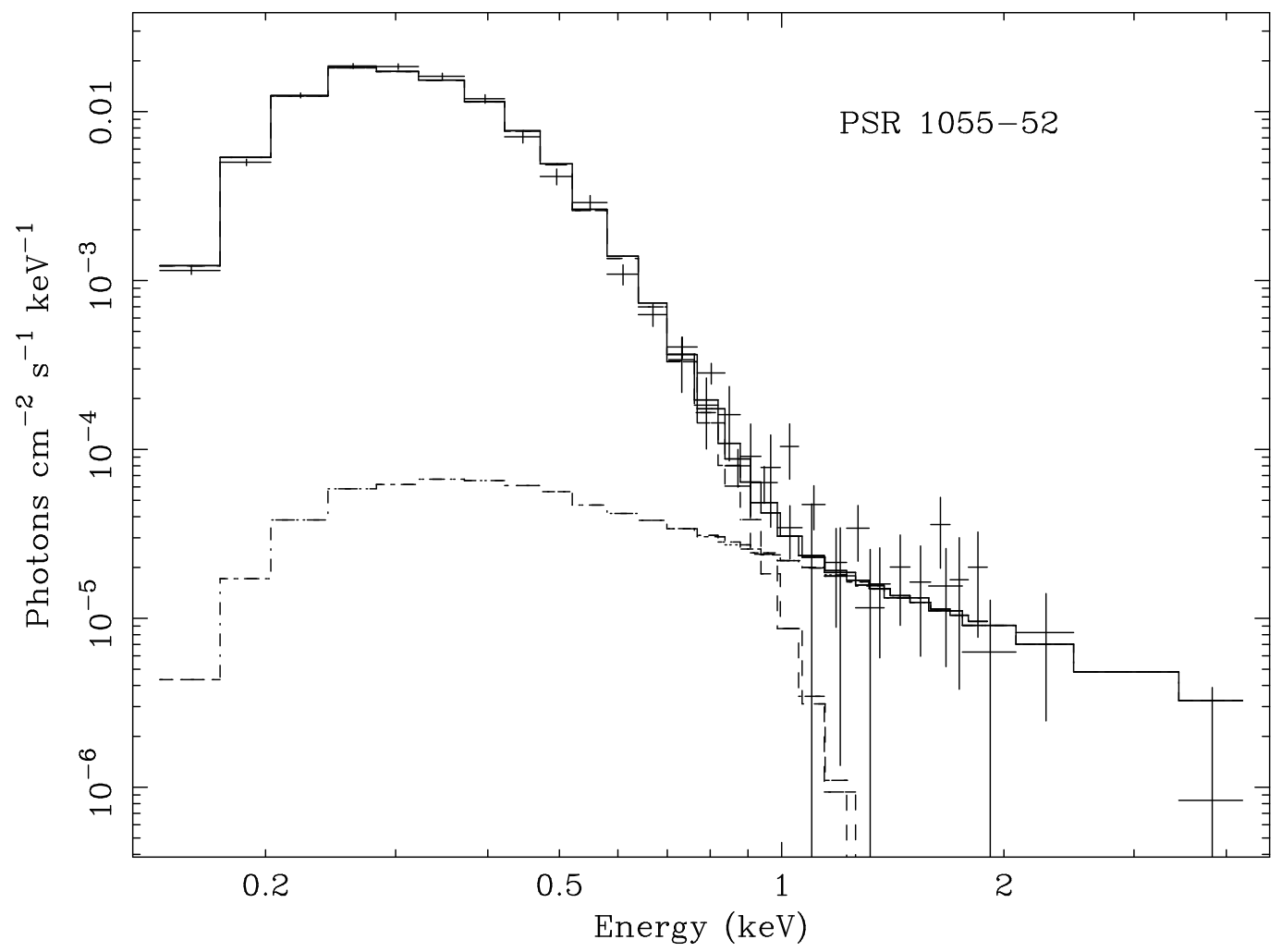

Fig. 2.- Fit of the blackbody plus power-law model to the $A S C A$ and ROSAT spectra of PSR 1055-52. 


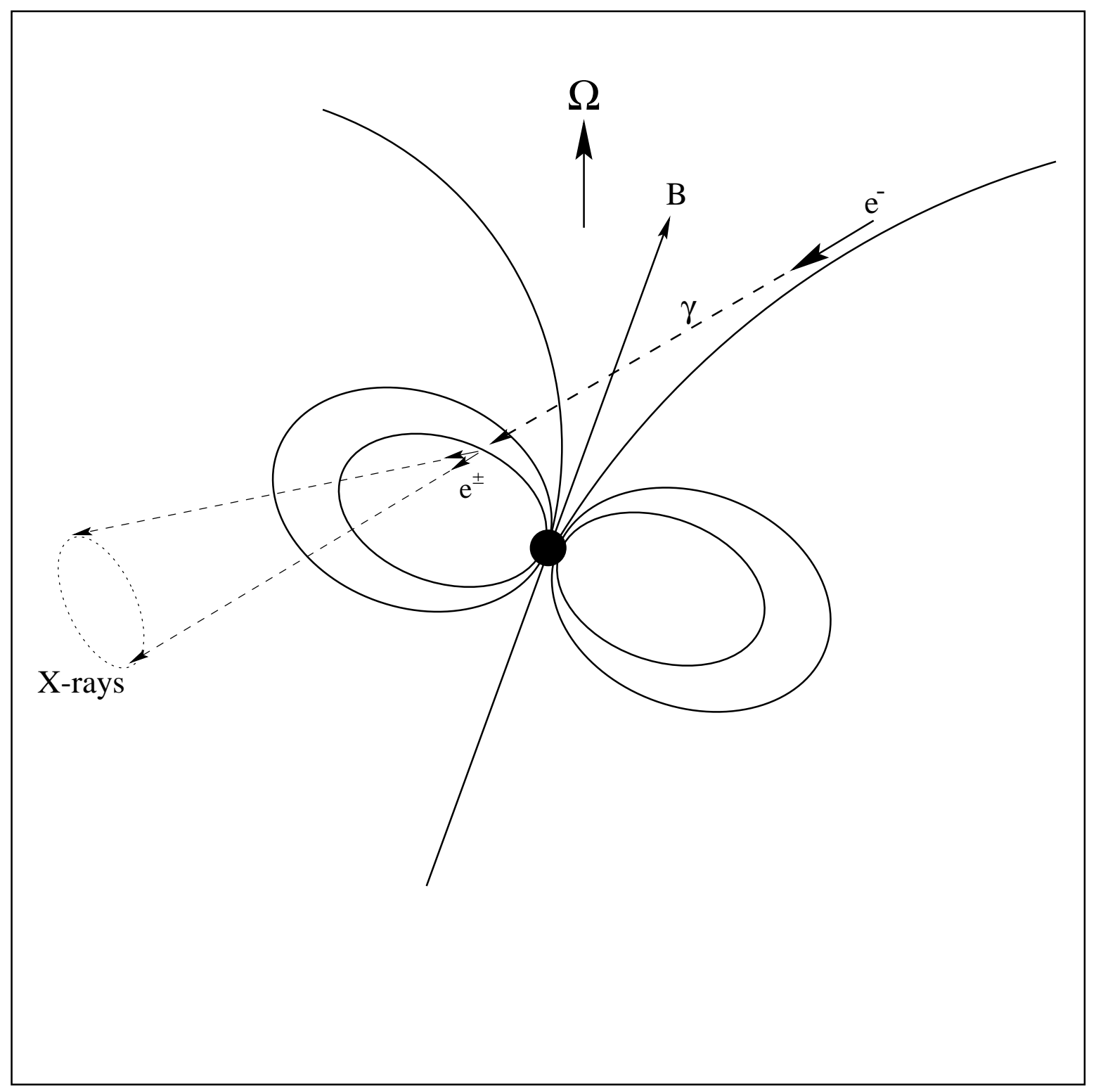

Fig. 3.- Curvature $\gamma$-ray photons from inflowing primary particles are converted to secondary $e^{ \pm}$pairs when passing through closed field lines. The $e^{ \pm}$pairs will then synchrotron radiate a power-law spectrum of energy index 0.5 . 

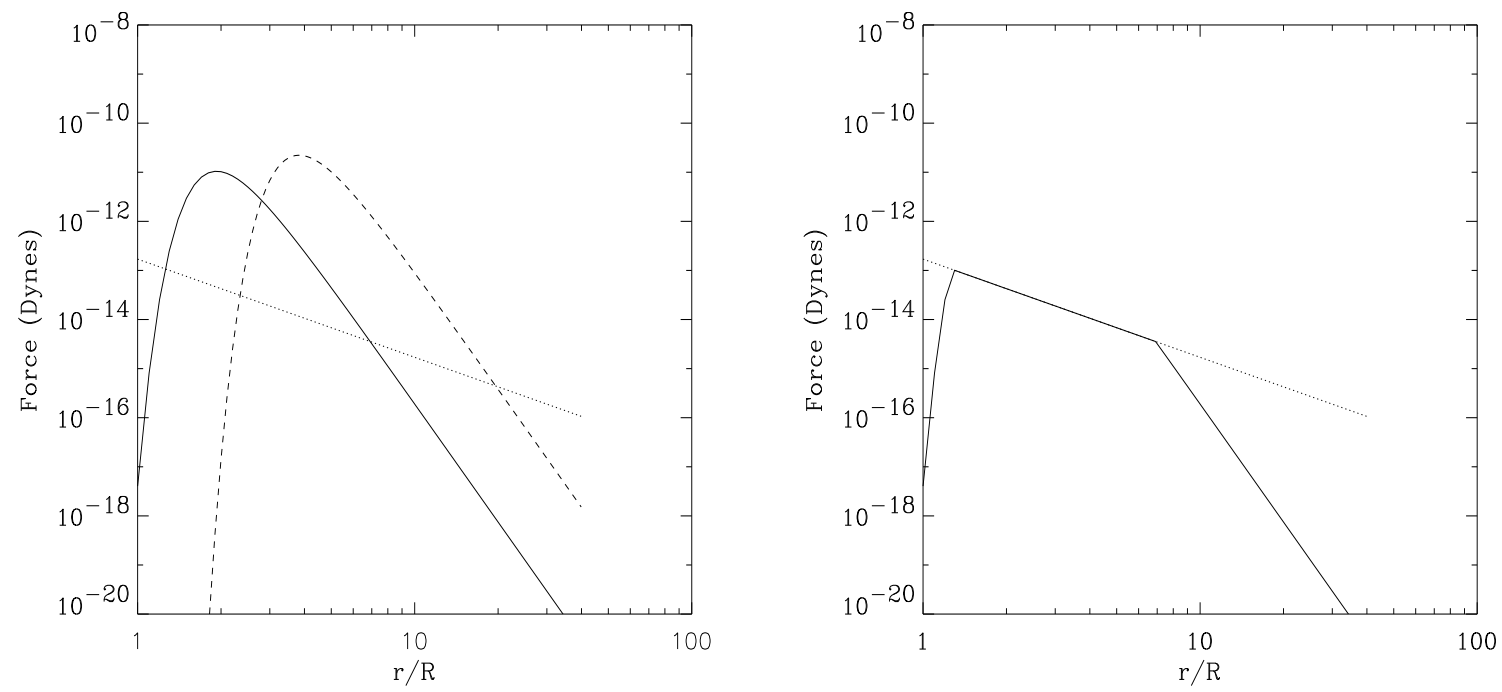

Fig. 4.- (a) The optically thin case for the radiation force and the gravitational force on $e^{ \pm}$as a function of distance $r$ from the center of the neutron star; the solid line indicates the radiation force for $k T=0.5 \mathrm{keV}$, the dashed line for $k T=0.06 \mathrm{keV}$. The dotted line gives the gravitational force. (b) The optically thick case for the radiation force and the gravitational force; these two forces are equal between the two equilibrium points. 


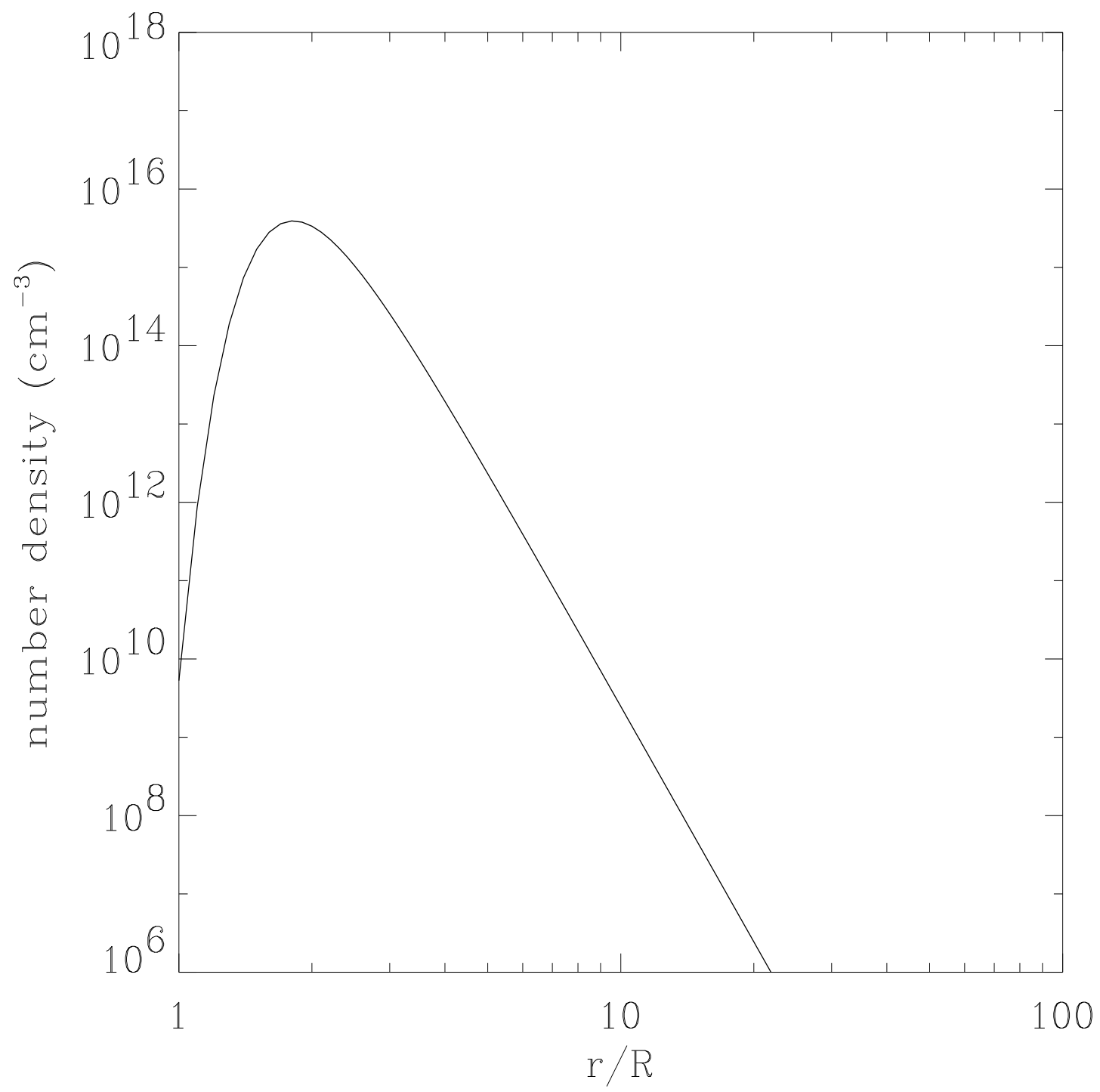

Fig. 5. - The maximum number density supportable by the radiation force as a function of distance $r$ for $k T=0.5 \mathrm{keV}$ and $L_{\mathrm{X}}=2.1 \times 10^{32} \mathrm{ergs} \mathrm{s}^{-1}$. 


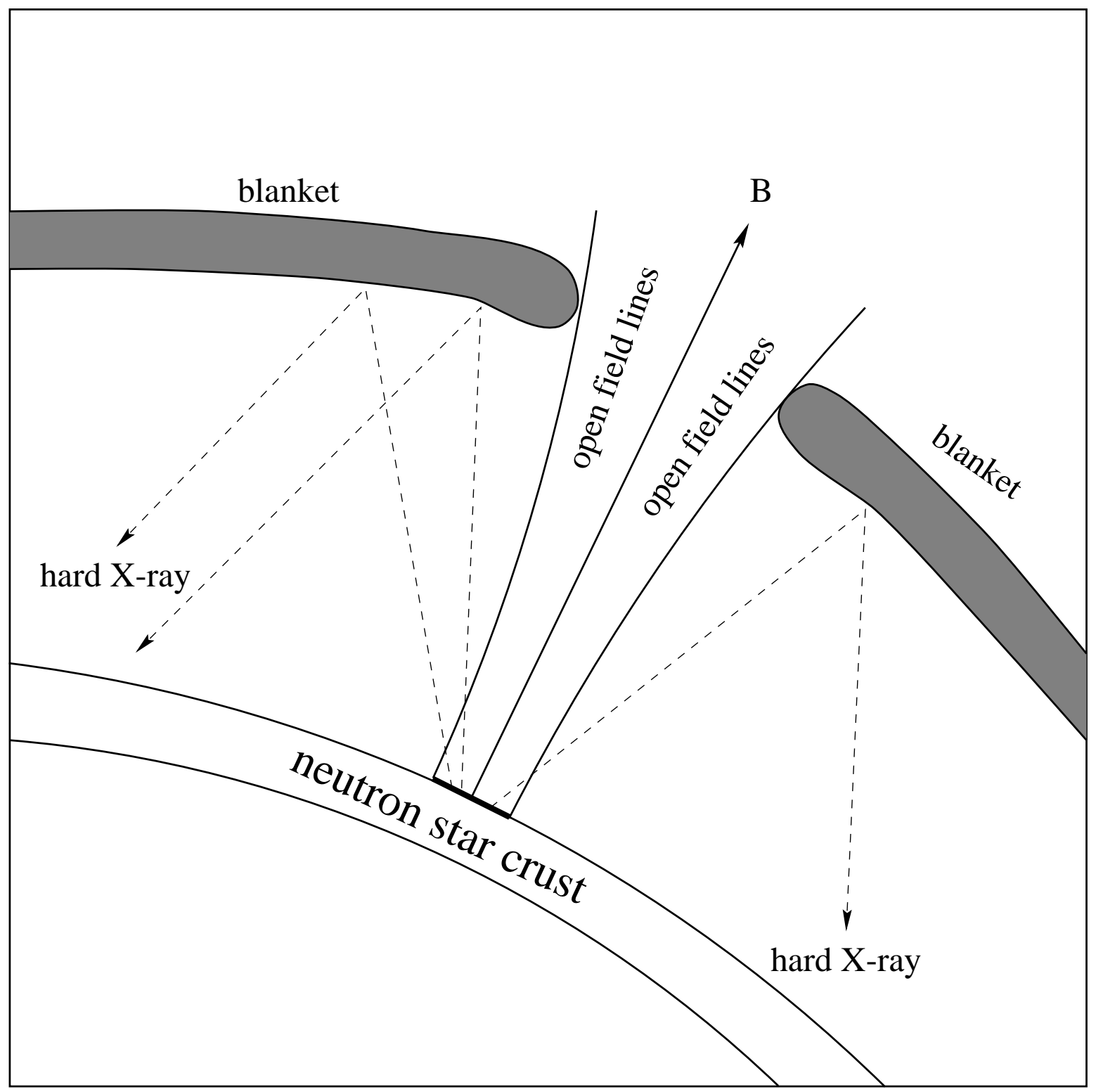

Fig. 6. - For $\gamma$-ray pulsars, the $e^{ \pm}$pairs created on closed field lines will form a reflecting blanket around the neutron star; hard X-rays from the heated polar caps will be reflected back to the surface of the star, and a direct observation of them is prevented. 

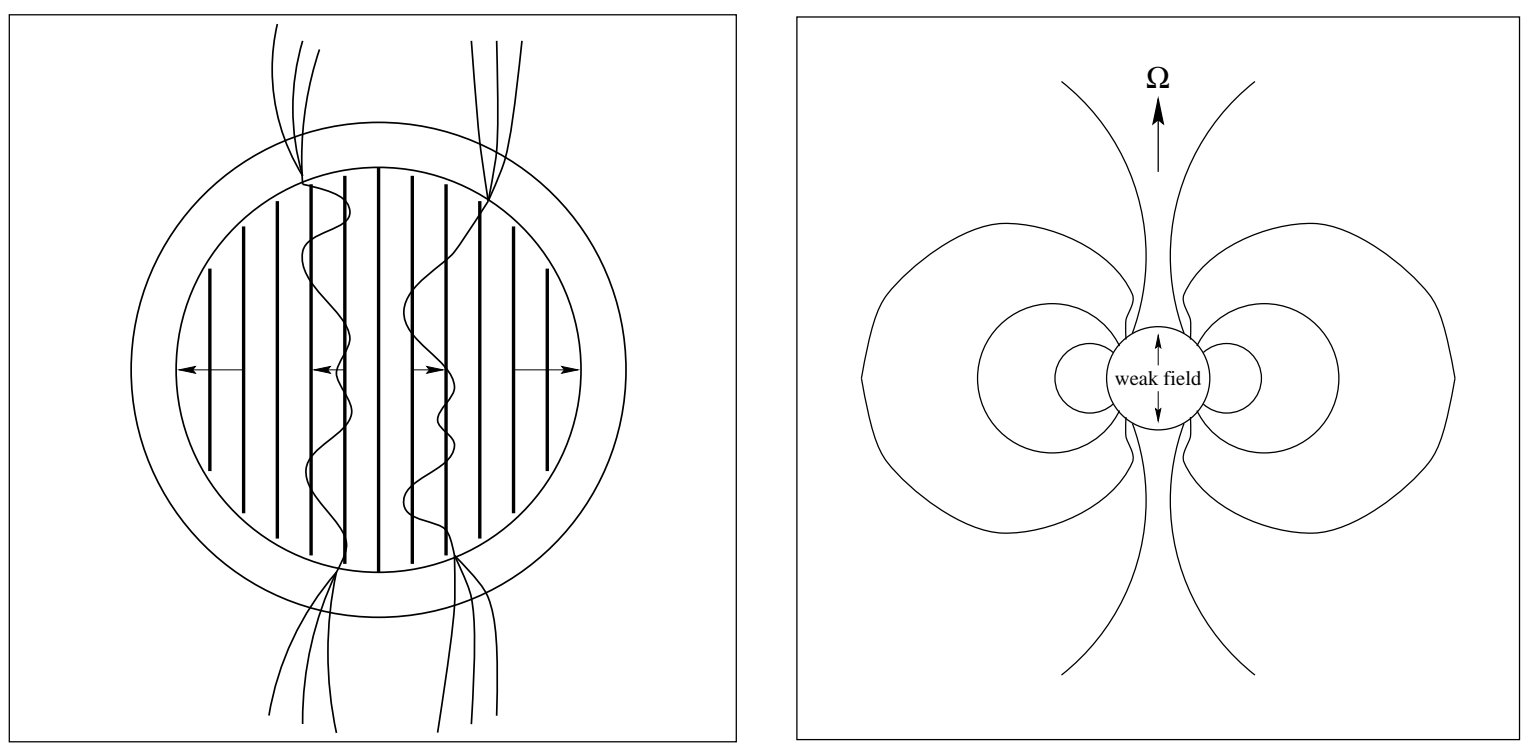

Fig. $\quad$ 7.- (a) The interior of a neutron star consists of superfluid neutrons and superconducting protons. The quantized superfluid vortex lines are parallel to the spin axis, but the superconducting flux tubes have a complicated twisted toroidal and poloidal structure. The superfluid vortex line array expands as the neutron star spins down. (b) The outward motion of vortex lines near the spin axis is slow enough to carry core flux tubes and crust magnetic field with these vortices and to leave a local region with anomalously weak magnetic field. The distorted field lines near the surface of the neutron star give a polar cap area larger than would be the case for a central dipole. 
Table 1. Pulsar Data

\begin{tabular}{|c|c|c|c|c|c|}
\hline & Geminga & PSR 1055-52 & PSR $0656+14$ & PSR $1929+10$ & PSR $0950+08$ \\
\hline$f(\mathrm{~Hz})$ & 4.218 & 5.073 & 2.598 & 4.414 & 3.951 \\
\hline$\dot{f}\left(\mathrm{~Hz} \mathrm{~s}^{-1}\right)$ & $-1.95 \times 10^{-13}$ & $-1.5 \times 10^{-13}$ & $-3.7 \times 10^{-13}$ & $-2.25 \times 10^{-14}$ & $-3.6 \times 10^{-15}$ \\
\hline$I \Omega \dot{\Omega}\left(\operatorname{ergs~s}^{-1}\right)$ & $3.3 \times 10^{34}$ & $3.0 \times 10^{34}$ & $3.8 \times 10^{34}$ & $3.9 \times 10^{33}$ & $5.6 \times 10^{32}$ \\
\hline$\tau($ year $)$ & $3.4 \times 10^{5}$ & $5 \times 10^{5}$ & $1.1 \times 10^{5}$ & $3.1 \times 10^{6}$ & $1.7 \times 10^{7}$ \\
\hline$B_{\mathrm{p}}\left(10^{12} \mathrm{G}\right)$ & 1.6 & 1.1 & 4.7 & 0.5 & 0.24 \\
\hline$T_{1}(\mathrm{~K})$ & $5.77 \times 10^{5}$ & $7.9 \times 10^{5}$ & $8.1 \times 10^{5}$ & - & - \\
\hline$L_{1}^{\mathrm{a}}\left(\operatorname{ergs~s}^{-1}\right)$ & $1.47 \times 10^{31}$ & $2.3 \times 10^{32}$ & $4.3 \times 10^{32}$ & - & - \\
\hline$\alpha^{\mathrm{b}}$ & $0.47_{-0.23}^{+0.25}$ & $0.5_{-0.3}^{+0.3}$ & - & - & - \\
\hline$T_{2}(\mathrm{~K})$ & - & - & $1.68 \times 10^{6}$ & $5.14 \times 10^{6}$ & $5.70 \times 10^{6}$ \\
\hline$L_{2}^{\mathrm{c}}\left(\operatorname{ergs~s}^{-1}\right)$ & $8.1 \times 10^{29}$ & $1.5 \times 10^{30}$ & $2.6 \times 10^{31}$ & $1.3 \times 10^{30}$ & $3.5 \times 10^{29}$ \\
\hline$A_{2}{ }^{\mathrm{d}}\left(\mathrm{cm}^{2}\right)$ & - & - & $5.7 \times 10^{10}$ & $3.2 \times 10^{7}$ & $5.8 \times 10^{6}$ \\
\hline$d(\mathrm{pc})$ & 160 & 500 & 400 & 250 & 125 \\
\hline
\end{tabular}

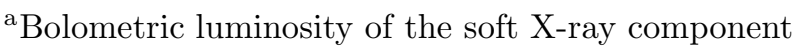

${ }^{\mathrm{b}}$ Power law energy index

${ }^{c}$ Luminosity of the hard X-ray component; $0.7-5.0 \mathrm{keV}$ for Geminga and PSR 1055-52, bolometric luminosity for PSR 0656+14, PSR 1929+10, and PSR 0950+08

${ }^{\mathrm{d}}$ Fitted area of polar cap

References. - Halpern \& Wang 1997; Greiveldinger et al. 1996; Wang \& Halpern 1997 